\title{
Fault Diagnosis Method Based on Modified Multiscale Entropy and Global Distance Evaluation for the Valve Fault of a Reciprocating Compressor
}

\author{
Ying Li - Jindong Wang* - Haiyang Zhao - Meiping Song - Lingfei Ou \\ Northeast Petroleum University, Mechanical Science and Engineering Institute, China
}

According to the nonlinearity, non-stationarity and multi-component coupling characteristics of reciprocating compressor vibration signals, a fault diagnosis method of a reciprocating compressor valve based on modified multiscale entropy (MMSE) and global distance evaluation (GDE) is proposed. First, the variational mode decomposition (VMD) method with superior anti-interference performance was utilized to analyse the strong non-stationarity vibration signals for all fault states. The modified multiscale entropy (MMSE) method provided for movingaverage procedures by replacing mean-average coarse-grained procedures was developed for the vibration signals after de-noising, and then the GDE method of overall parameter selection was introduced to evaluate the extracted MMSE and to select the optimal sensitivity scale feature. Finally, a binary tree of support vector machine (BTSVM) was selected as the classifier to identify the reciprocating compressor valve fault type. By analysing the experimental data, it can be shown that the method can effectively identify the fault type of the reciprocating compressor valve.

Keywords: fault diagnosis, reciprocating compressor valve, modified multiscale entropy, global distance evaluation, binary tree of support vector machine

Highlights

- $\quad$ An integrated fault feature extraction method of a reciprocating compressor valve based on the VMD-MMSE and GDE is proposed.

- A consistent number $K$ of band-limited intrinsic mode functions (BLIMFs) were selected based on a novel criterion for all fault states

- $\quad$ The MMSE method provided for the moving-average procedure by replacing mean-average coarse-grained procedure was developed for the vibration signals, GDE was introduced to refine the eigenvectors for higher recognition efficiency and accuracy.

- The effectiveness of this method is verified by the recognition results of BTSVM in comparison to other feature extraction methods.

\section{INTRODUCTION}

A reciprocating compressor is frequently used in the petroleum and petrochemical industries, its structure is complex, and multiple reasons for failure exist, of which more than $60 \%$ occur in the valve. The ability to quickly and accurately find and diagnose the fault type for reciprocating compressor fault diagnosis is of great significance [1]. Due to the nonlinearity, non-stationarity, and multi-component coupling characteristics of reciprocating compressor valve vibration signals, using the traditional linear theory of signal analysis methods for fault diagnosis has more limitations, and it is difficult to effectively extract fault features. Consequently, these feature extraction methods based on nonlinear dynamic parameters, such as fractal dimension, approximate entropy [2], and sample entropy [3], are gradually being introduced to the field of fault diagnosis. These methods can describe the nonlinear characteristics of vibration signals from different perspectives, but merely reflect the characteristics of vibration signals from a single scale of information.

Multiscale entropy (MSE) is used by Costa et al. [4] and [5] and others to propose a nonlinear characteristics analysis method; it is a measurement method of the complexity and the random degree of time series under different scale factors. In the literature [6] to [8], the MSE method is used to quantify and extract the characteristics of vibration signals in the fault diagnosis of rolling bearings and gearboxes. The results show that MSE can describe the complexity of time series and effectively distinguish the types of faults. However, when different time scales are derived by calculating the coarse-grained time series, the length of the time series will be shortened as the scale factor increases, resulting in an imprecise estimation of entropy or undefined entropy when the scale factor is larger, and the endpoint "Flying wing" phenomenon occurs. Therefore, this paper proposes replacing the commonly used meanaverage coarse-grained procedure with a moving- 
average coarse-grained procedure, which not only avoids the phenomenon of losing data after a coarsegrained procedure, but also greatly improves the accuracy of the algorithm.

Aiming at the nonlinearity, non-stationarity, and multi-component coupling characteristics of reciprocating compressor valve vibration signals, it is feasible to calculate the modified multiscale entropy (MMSE) for the characteristic state description of reciprocating compressor valve vibration signal. The vibration signal of a reciprocating compressor valve contains a large number of random noise components; however, they are susceptible to the influence of the noise signal; when simply calculating multiscale entropy to process the vibration signal of a reciprocating compressor valve, it will directly influence the outcome of feature extraction. Therefore, it is necessary to pre-process the original signal for reducing or eliminating the noise disturbance before a further analysis [9] to [11]. Zhao et al. [12] and [13] applied the improved local mean decomposition (LMD) to process the different bearing clearance fault states of a reciprocating compressor. Multiscale morphological filtering (MMF) was put forward by $\mathrm{Li}$ et al. [14] to pre-process the vibration signals of planetary gearboxes before the fault extraction. Zhang et al. [15] and $\mathrm{Bi}$ et al. [16] utilized the empirical mode decomposition (EMD) and frequency modulated empirical mode decomposition (FM-EMD) to pre-process the vibration signals of a gearbox with different faults in diagnosing and monitoring the conditions of the gearbox. In addition, ensemble empirical mode decomposition (EEMD) and other methods were used to pre-process the vibration signals [17] and [18].

The variational mode decomposition (VMD) method [19] and [20] is an adaptive signal processing method, successfully utilized for extracting the fault features for nonlinear and non-stationary rub-impact signals [21] to [23], the instantaneous detection of speech signals [24] and trends analysis of financial markets [25]. Therefore, a feature extraction algorithm in combination with VMD and MMSE can improve the accuracy of feature extraction.

For the MMSE eigenvalues, when the fault type and data are larger, the classification sensitivity and classification level of MMSE corresponding to the characteristics of different scales are different; if only to the MMSE eigenvalues are used to identify the fault type, the failure classification accuracy and precision are relatively low, it is necessary to optimize the MMSE eigenvalues. Therefore, a novel global distance evaluation (GDE) method is proposed to solve the problem of the optimal parameters.

This paper combines the VMD-MMSE with the GDE algorithm, and proposes a fault feature extraction method based on the VMD-MMSE and GDE. The method was applied to the fault feature extraction of reciprocating compressor valve vibration signals, used to analyse different forms of valve failure, and a binary tree of support vector machine (BTSVM) was applied to different fault classification of valve fault, which provided a new method of fault diagnosis for a reciprocating compressor valve.

\section{MMSE AND GDE PRINCIPLES}

\subsection{Multiscale Entropy}

Sample entropy is the complexity and lack of rules of time series on a single scale. When sample entropy is gradually reduced, the similarity of the time series increases. When sample entropy increases gradually, the complexity of the time series will be higher. For multiscale entropy, it is on the basis of sample entropy; therefore, multiscale entropy shows the sample entropy of time series under different scales, reflecting the degree of complexity of the time series in different scales with the sequence indicated in multiple scales containing more information [6]. Therefore, in the analysis of multiscale entropy, measuring the complexity of time series should be chosen by sample entropy for analysis. The calculation process of multiscale entropy is as follows:

(1) To obtain the coarse-grained original time series, and construct time series at different time scales. The coarse-grained time series is $\left\{q_{j}^{(\tau)}\right\}$, calculated according to the following formula:

$$
q_{j}^{(\tau)}=\frac{1}{\tau} \sum_{i=(j-1) \tau+1}^{j \tau} q_{i}, \quad(1 \leq j \leq N / \tau, \tau=1,2, \ldots, N) .
$$

(2) The time series is $\{x(i), i=1,2, \ldots, N\}$, calculated its SampEn and selected model dimension for $\mathrm{m}$, similar tolerance for $r$, computing as follows [6]:

(a) Set time series to a set of $m$ dimensional vector:

$$
\begin{aligned}
X(i)=\{x(i), x(i+1), \ldots, x(i+m-1), & (i=1,2, \ldots, N-m+1) .
\end{aligned}
$$

(b) The definition of the distance between $X(i)$ and $X(j)$ is $d[X(i), X(j)]$, which is one of the biggest in the corresponding element, namely

$$
d[X(i), X(j)]=\underset{k=0 \rightarrow m-1}{\operatorname{Max}}\{|X(i+k)-X(j+k)|\} .
$$


(c) For $i$, statistics the number of $d[X(i), X(j)]<r$ is $n_{i}^{m}, i=1,2, \ldots, N-m+1, j=1,2, \ldots, N-m+1$, and $j \neq i$, then calculate the ratio of it and the total distance $N-m$ (template matching number), and namely:

$$
C_{i}^{m}(r)=\frac{n_{i}^{m}}{N-m},
$$

while the mean of $C_{i}^{m}(r)$ is:

$$
C^{m}(r)=\frac{1}{N-m+1} \sum_{i=1}^{N-m+1} C_{i}^{m}(r)
$$

(d) When $m=m+1$, repeat steps (a) to (c), statistics $C^{m+1}(r)$.

(e) Calculation formula of the final SampEn is

$$
\text { SampEn }=-\ln \frac{C^{m+1}(r)}{C^{m}(r)} .
$$

With the calculation formula of sample entropy, it can be seen that the size of the sample entropy depends on $m, \quad r$, usually $m \in[2,10]$, $r \in[0.1,0.5] S D$.

(3) Multiscale entropy is the function of the scale factor $\tau$ as independent variables, SampEn as dependent variable.

$$
\operatorname{MSE}(\tau)=\operatorname{SampEn}(X(\tau), m, r) .
$$

\subsection{Modified Multiscale Entropy}

The traditional coarse-grained method is to compress the original time series by the scale factor $\tau$ in turn. Since the original time series is a finite amount of data, as the scale factor $\tau$ gradually increases, the length of the coarse-grained time series decreases. While the original data length is not an integer multiple of the scale factor $\tau$, it will result in the loss of partial data, and the emergence of the "flying wing" phenomenon, affecting the accuracy of subsequent algorithms. Therefore, a coarse-grained method based on the sliding average method is proposed for the coarsegrained original time series, which not only avoids the phenomenon of data loss after the coarse-grained series but also greatly improves the accuracy of the algorithm.

(1) The proposed MMSE method is as follows: Let $P_{j}^{(\tau)}$ represent the moving-averaged time series at a scale factor $\tau$, calculated according to the following formula:

$$
p_{j}^{(\tau)}=\frac{1}{\tau} \sum_{i=j}^{j+\tau-1} x_{i},(1 \leq j \leq N-\tau+1)
$$

(2) The modified multiscale entropy of the time series after calculating the average coarse-grained time series is calculated:

$$
\operatorname{MMSE}(\tau)=\operatorname{SampEn}\left(p^{(\tau)}, m, r\right) .
$$

\subsection{Comparison between MMSE and MSE Simulation}

\subsubsection{Simulation Signal Analysis}

In order to compare the MMSE and the MSE, respectively for different lengths of $1 / f$ noise and Gaussian white noise signals are analysed, both the time domain waveform are shown in Fig. 1.

Using MSE and MMSE to analyse length $N=$ 2048, 4096, 6144, 8192 and 10240 of $1 / f$ noise and Gaussian white noise signals, respectively, shown in Fig. 2, which $m=2, r=0.15 \mathrm{SD}$.
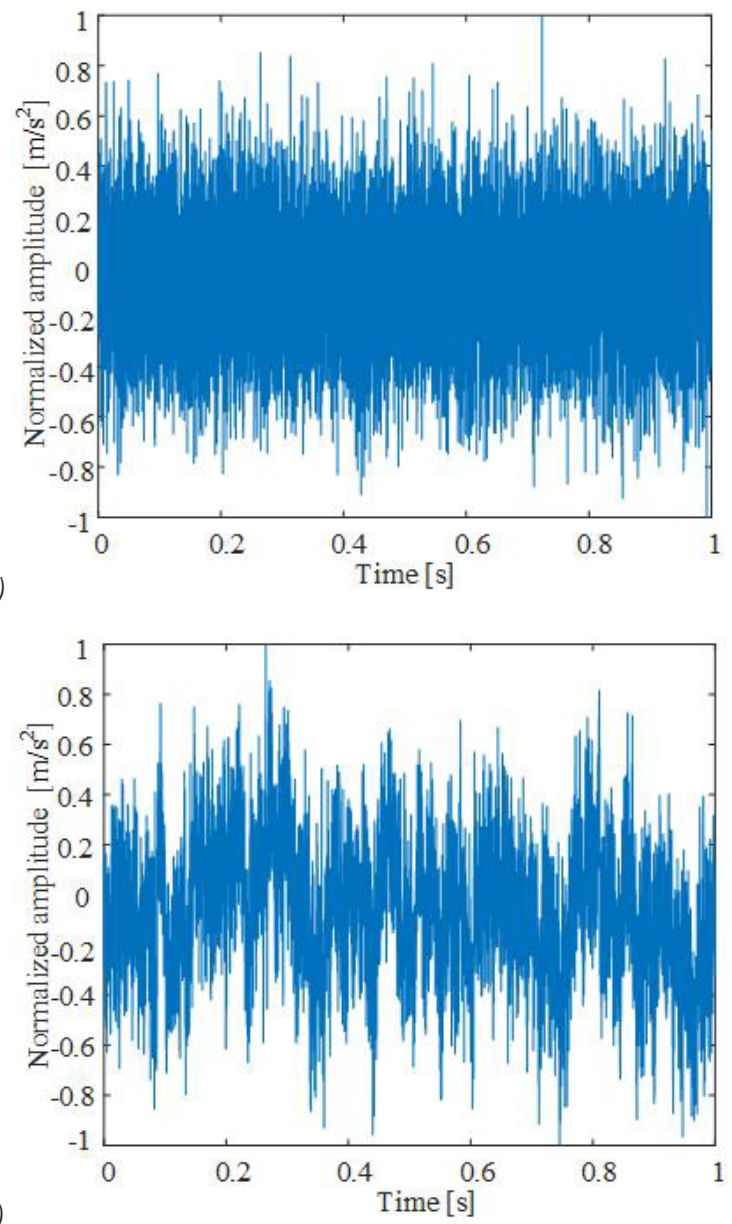

Fig. 1. Gaussian white noise signal and $1 / f$ noise signal waveform; a) Gaussian white noise signal, b) $1 / f$ noise signal 

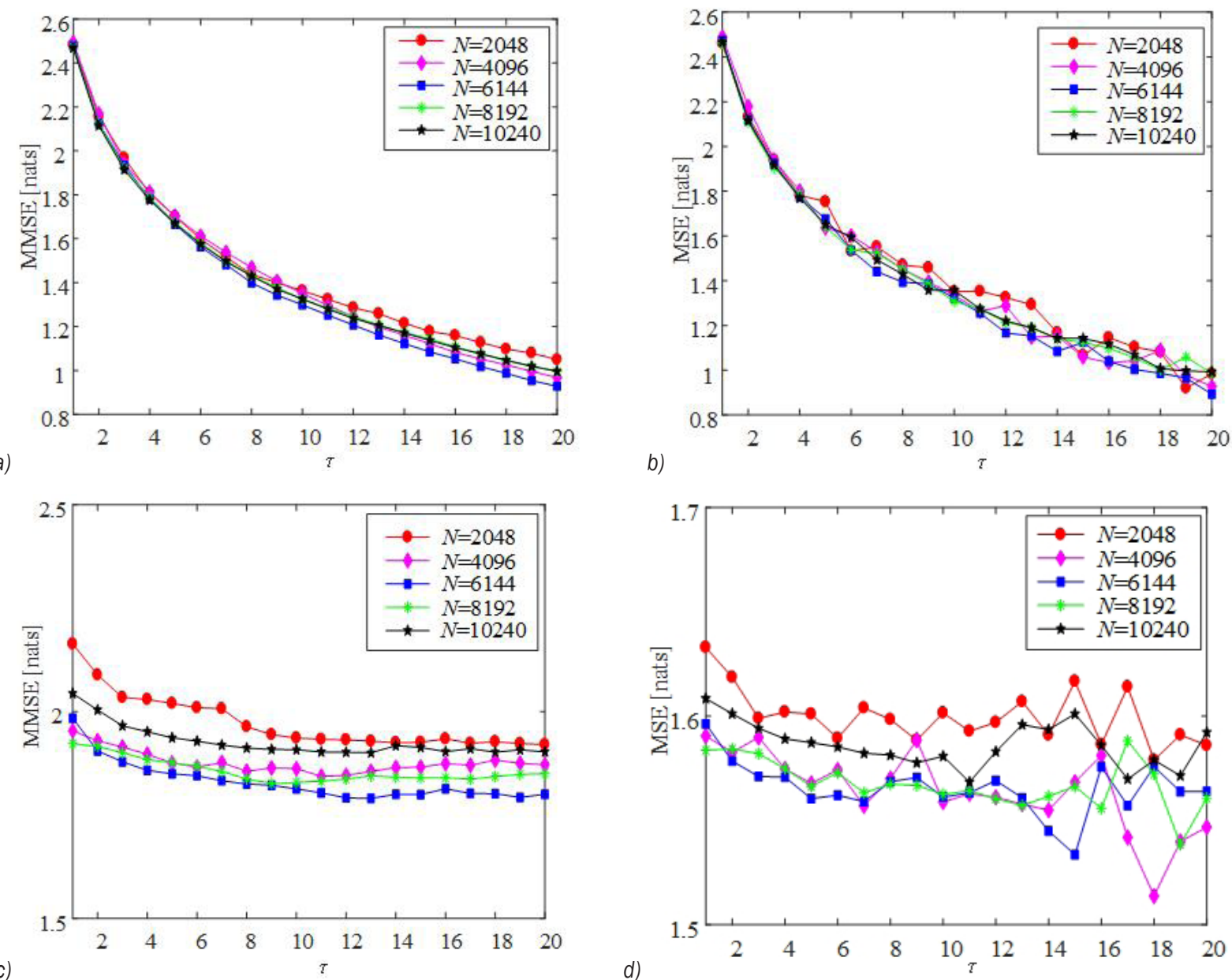

Fig. 2. MMSE and MSE curves of different lengths of white noise and 1/f noise; a) MMSE with different lengths of white noise, b) MSE with different lengths of white noise, c) MMSE with different lengths of $1 / f$ noise, $d$ ) MSE with different lengths of $1 / f$ noise

As can be seen from Fig. 2, firstly as the scale factor $\tau$ increases, the MSE and MMSE curves of white noise gradually decrease, the MSE and MMSE curves of $1 / f$ noise tend to be constant, but the MSE curves of white noise and $1 / f$ noise increase with the scale factor $\tau$, specifically on a larger scale; the entropy fluctuation range is relatively large, while the MMSE curve changes more gradually with the increase of the scale factor. This shows that as the scale factor $\tau$ increases, the MMSE has a better consistency than the MSE to obtain a stable entropy value; secondly, when the data length $\mathrm{N}$ is greater than 2048, the MSE or the MMSE curves of different lengths of data have only a slight difference. Furthermore, the MMSE (or MSE) curve of the white noise decreases as the scale factor increases, indicating that the white noise signal contains important feature information only in the coarse-grained sequence with smaller scale; whereas the MMSE (or MSE) of the $1 / f$ noise is small with a large change in the scale factor, and it tends to be almost stationary, and the entropy of the $1 / f$ noise is greater than the entropy of the white noise in most scales. This shows that $1 / f$ noise contains rich feature information and has more complex structural characteristics than white noise signals do.

To study the statistical stability effects of the MSE and the MMSE, the MSE and the MMSE analysis was performed on white noise and $1 / f$ noise signals, and 200 groups of independent noise data were randomly generated. Each group of data contained 1000 data points. The simulation results are shown in Fig. 3.

For the white noise shown in Fig. 3a, the mean values of the entropy calculated using the MSE and the MMSE are almost equal, but the standard deviation of MMSE is much smaller than the standard deviation of MSE, which shows that the MSE and 


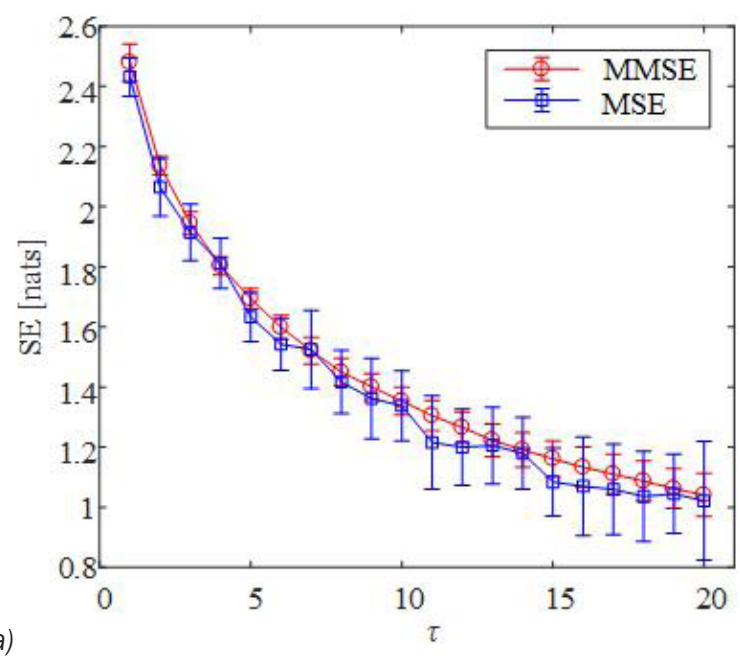

a)

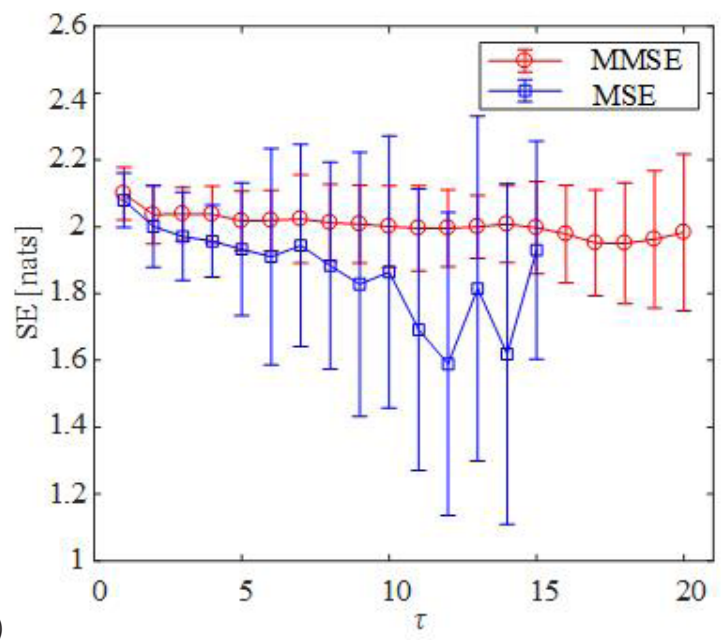

Fig. 3. Comparative analysis of the MMSE and the MSE; a) white noise signal, b) $1 /$ f noise signal

the MMSE methods are almost statistically stable. However, the MMSE method provides more accurate entropy estimation than the MSE method does. For the $1 / f$ noise shown in Fig. 3b, the MSE method causes undefined entropy when the coarse-grained scale is greater than 16, and the MMSE method calculates the entropy value for all coarse-grained scales. By comparing it with the MSE method, it can be seen that the proposed MMSE method is more effective in extracting different structural characteristics of white noise and $1 / f$ noise and providing more accurate entropy estimation.

\subsubsection{Valve Analog Signal Analysis}

For the four states of the valve, 20 groups of twowhole-period data were selected, and the analysis methods in Section 1.3.1 were adopted to conduct MSE and MMSE analysis respectively, in which $m=2, r=0.15 \mathrm{SD}$. The results are shown in Figs. 4 and 5. As can be seen from Fig. 4, as the scale factor $\tau$ increases, most of the MSE and MMSE curves of the four reciprocating compressor valve states gradually decrease, but only $\tau \leq 3$, the MSE and MMSE curves increase. The larger the entropy values, the more stable the curves are. However, the MSE curves of the four reciprocating compressor valve states increase with the scale factor $\tau$; specifically on a larger scale, the entropy fluctuation range is relatively large, while the MMSE curve changes more gradually with the increase of the scale factor. It also turns out that the MMSE has a better consistency than the MSE to obtain a stable entropy value as the scale factor $\tau$ increases.
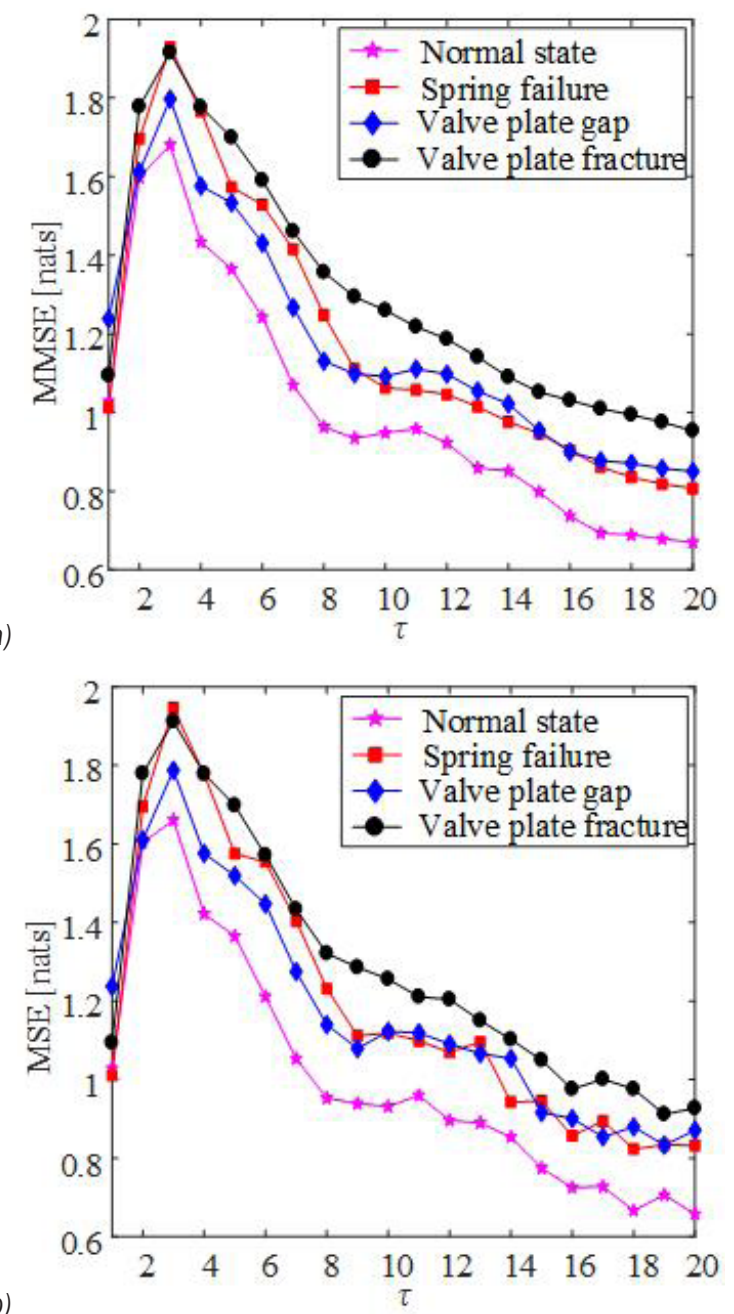

Fig. 4. MMSE and MSE curves of different valve states; a) MMSE with different valve states, b) MSE with different valve states 
Similarly to studying the statistical stability effects of the MSE and the MMSE for the four reciprocating compressor valve states, the MSE and the MMSE analysis was performed on 20 groups of the four valve states data were randomly generated. Each group of data contained two whole-period data points. The calculation results are shown in Fig. 5.

For the four valve states shown in Fig. 5, the mean values of the entropy calculated using the MSE and the MMSE are almost equal, but the individual values of the MSE and the MMSE is discrepant; in particular, the larger the scale $\tau$, the greater the discrepancy and obvious fluctuation. Furthermore, the standard deviation of MMSE is much smaller than the standard deviation of MSE, which shows that the MSE and the MMSE methods are almost statistically stable. However, the MMSE method provides more

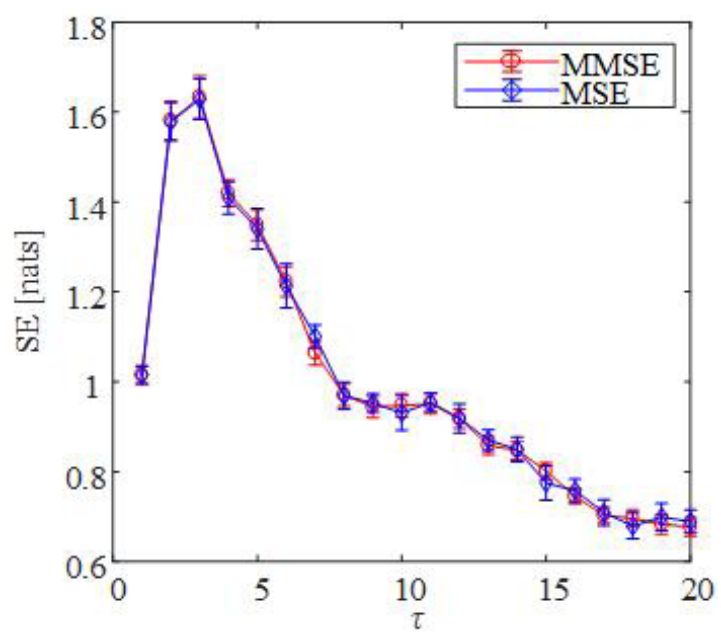

a)

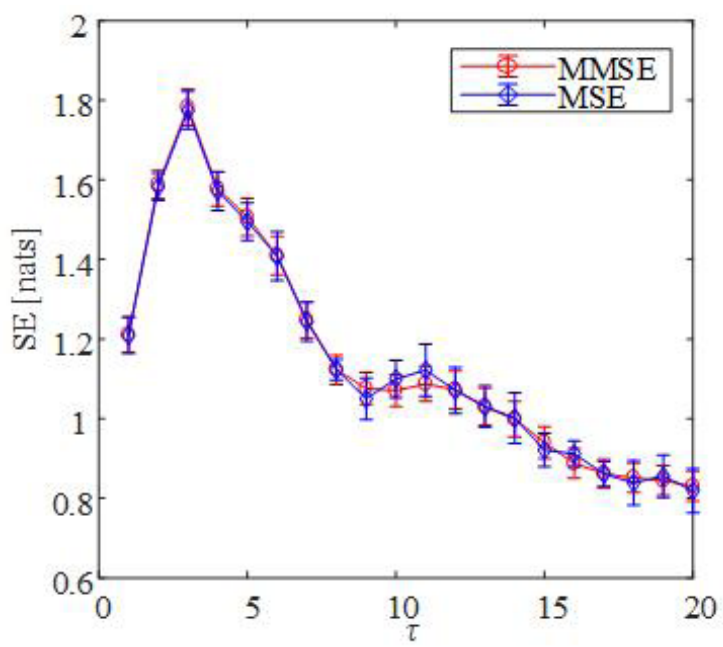

accurate entropy estimation than the MSE method does. It can be seen that the proposed MMSE method is more effective in extracting the different structural characteristics of the four valve states and providing more accurate entropy estimation.

\subsection{Global Distance Evaluation Method}

According to the vibration signals under different conditions, extracting their MMSE eigenvalues, of which the vibration signal under different conditions and different scales as a feature vector, yields original MMSE feature set $T$, namely:

$$
\begin{gathered}
\{T \mid \operatorname{MMSE}(l, m, n)\}, \\
l=1,2, \ldots, L ; m=1,2, \ldots, M ; n=1,2, \ldots, N,
\end{gathered}
$$

b)
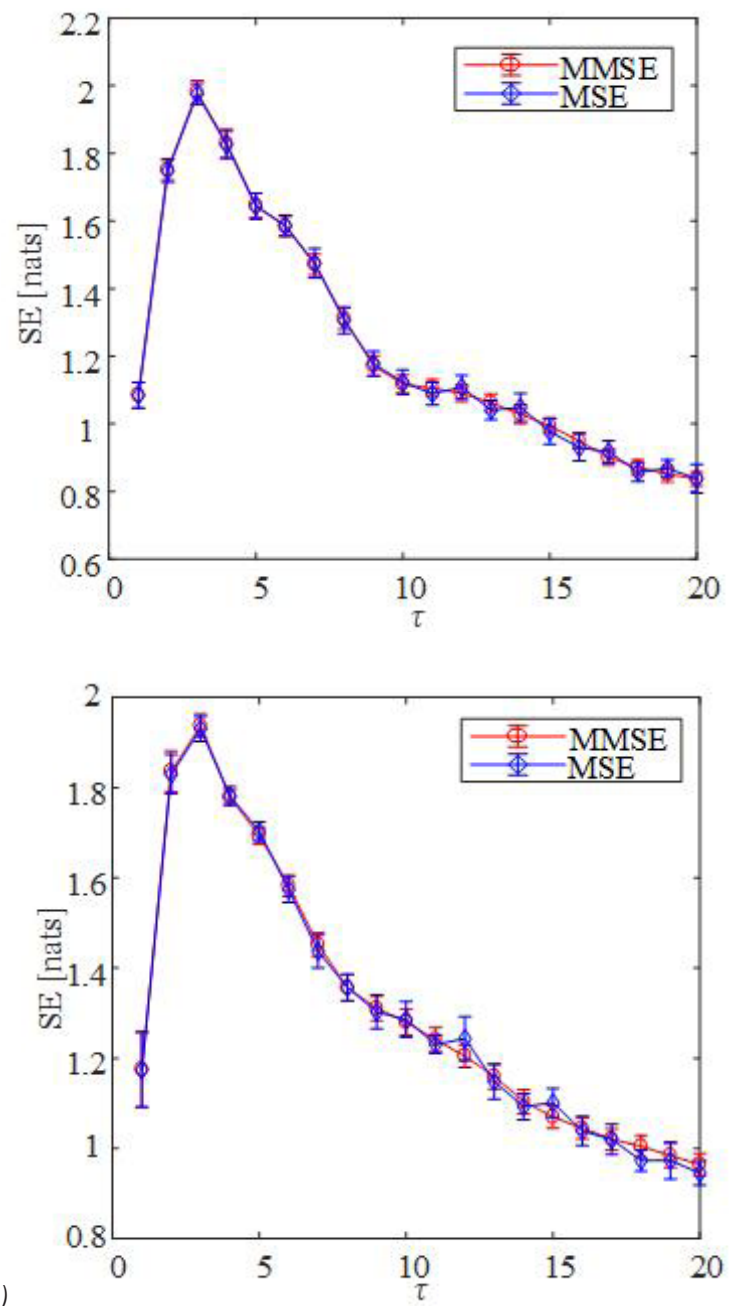

Fig. 5. Comparative analysis of the MMSE and the MSE for the four reciprocating compressor valve states; a) normal state, b) spring failure, c) valve plate gap, d) valve plate fracture 
where $\operatorname{MMSE}(l, m, n)$ denotes the sample entropy feature of $l^{\text {th }}$ scale of $m$-category under $n^{\text {th }}$ sample; $l$ is number of scale; $M$ is the state class number; $N$ is a sample number.

The MMSE feature set $T$ contains a larger amount scale of the feature vector, generally not less than 10 . Under different conditions and different scales, fault feature vector set $T$ reflects the sensitivity of different degree, and redundancy between them still exists. To improve the classification performance and efficiency of feature vector set $T$, this paper introduced the global distance evaluation technique to MMSE original feature set $T$ of Eq. (10) for optimal selection feature, specific steps are as follows:

(1) The average distance within the class of similar state samples

$$
\begin{aligned}
& d_{l, m}=\frac{1}{N(N-1)} \sum_{m, n=1}^{N}|\operatorname{MSE}(l, m, n)-\operatorname{MSE}(l, m, q)|, \\
& n, q=1,2, \ldots, N ; q \neq n, m=1,2, \ldots, M ; l=1,2, \ldots, L .
\end{aligned}
$$

Among, $\operatorname{MMSE}(l, m, n), \quad \operatorname{MMSE}(l, m, z)$ respectively denotes entropy value of $n^{\text {th }}$ sample and $z^{\text {th }}$ sample under $I^{\text {th }}$ scale of m-category. Therefore, the average distance class under the same scale $I$ of $m$ classes is:

$$
D_{l}=\frac{1}{M} \sum_{m=1}^{M} d_{l, m}
$$

(2) The average distance between different class states

$$
D_{l}^{\prime}=\frac{1}{M(M-1)} \sum_{u, v=1}^{M}\left|M S E^{\prime}(l, u)-M S E^{\prime}(l, v)\right|
$$

Among, $\operatorname{MMSE}^{\prime}(l, u), \operatorname{MMSE}(l, v)$ denotes the average value of $\mathrm{m}$ samples entropy under $l^{\text {th }}$ scale of $u^{\text {th }}$ and $v^{\text {th }}$ class.

Define sensitivity

$$
\varphi_{l}=D_{l}^{\prime} / D_{l} .
$$

$\varphi_{l}$ described the $l_{\text {th }}$ scale feature of sensitivity of classifying M class, $\varphi_{l}$ is larger, the more sensitive features, more easily classified.

(3) Determine the restrictive conditions, namely

$$
\left|\varphi_{\text {mean }} / \varphi_{\text {mean }}\right|>t h,
$$

among, $\varphi_{\text {mean }}$ for average sensitivity; th for the threshold.

The scale features above conditions are selected as the sensitive features. Constitute the sensitive feature subset $T 1$, and send it to BTSVM for the classification comparison.

\section{FAULT DIAGNOSIS METHOD BASED ON MMSE AND GDE}

A novel feature extraction method based on the MMSE and GDE is proposed, and its application for reciprocating compressor valve fault diagnosis is described in Fig. 6. The method decomposes vibration signal by VMD, and then chooses a series of band-limited intrinsic mode functions (BLIMF) components including the main components of the fault information to reconstruct signal, quantitatively described by MMSE and GDE, and finally adopts BTSVM to identify fault type. The specific steps are as follows:

(1) For the vibration signals of different fault states, we employ the VMD method to decompose each signal into $K$ number of BLIMFs, and select adaptively a uniform $K$ number BLIMFs of VMD decomposition for all fault states.

Initialization modal number $K=2$, penalty factor and bandwidth values are $\sigma=0, \alpha=2000$, respectively. Observing the central frequency of each BLIMF component after decomposition, if the central frequencies are similar, the modal number $K=K-1$ is determined, whereas the modal number $K=K+1$, repeated the above process until the centre frequency is similar. Calculate the relationship between each BLIMF component and the vibration signal, and determine the BLIMF components with the main information, and reconstruct the signal.

(2) Calculate the MMSE of each type of the reconstruction signal, and form a feature set of MMSE.

(3) Using the GDE method to select the feature optimization of MMSE feature set, obtain the sensitive feature set of MMSE.

(4) With BTSVM as a pattern recognition classifier, send the sensitive feature set of MMSE to BTSVM for the classification comparison and identify fault type.

\section{FAULT DIAGNOSIS FOR RECIPROCATING COMPRESSOR VALVE BASED ON MMSE AND GDE METHOD}

In this study, the proposed MMSE and GDE method is utilized to extract the fault feature of the valve vibration data from a two-stage double-acting reciprocating compressor of type (2D12), as shown in Fig. 7. The shaft power of reciprocating compressor is $500 \mathrm{~kW}$, the piston stroke is $240 \mathrm{~mm}$, and the motor speed is $496 \mathrm{rpm}$. The valve is one of the core components of the reciprocating compressor; its safe and stable operation is of great significance. 


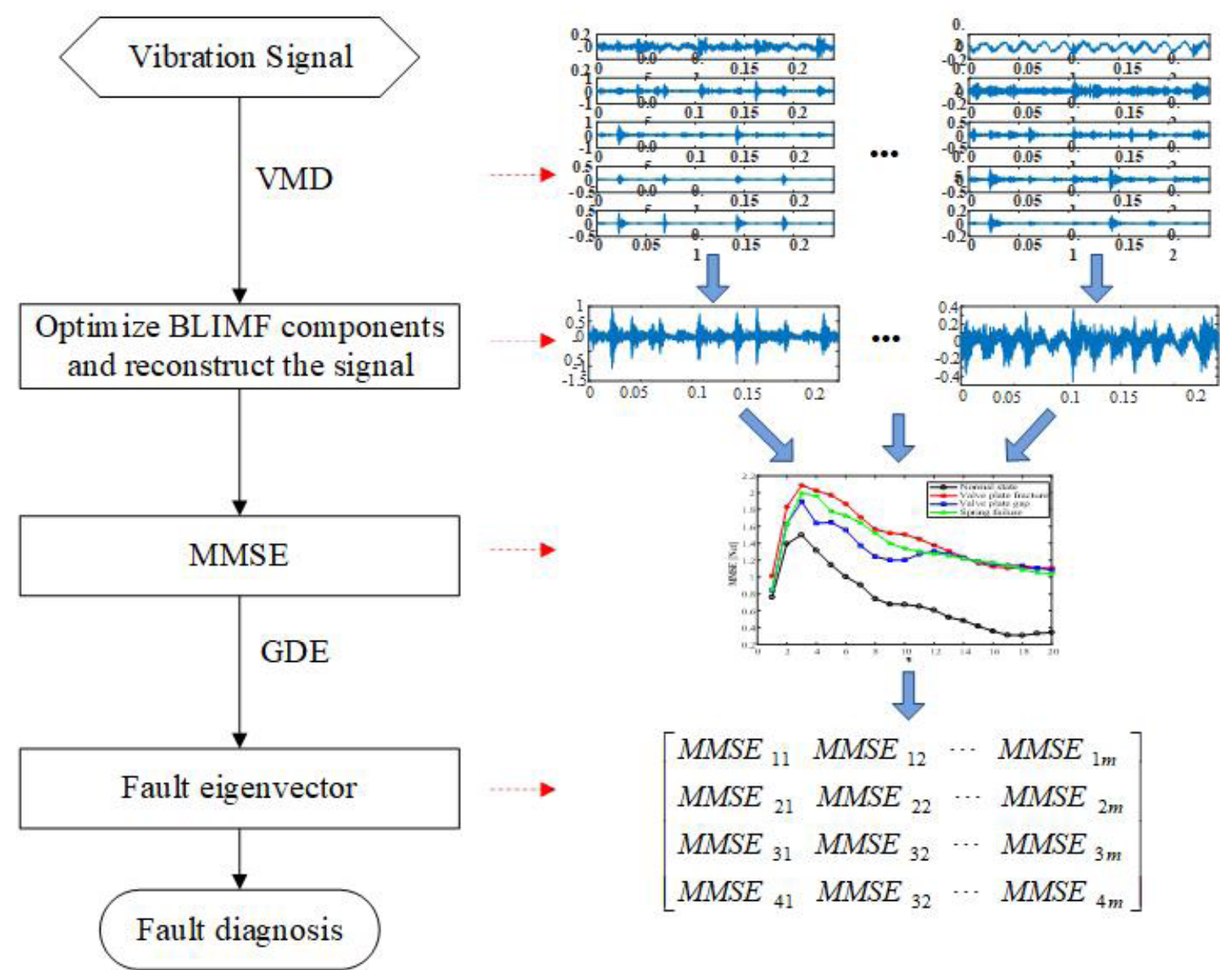

Fig. 6. Flowchart of fault diagnosis algorithm based on MMSE and GDE

The 2D12 reciprocating compressor valve is a ring valve that is composed of a valve seat, a valve plate, a valve lift limiter, a spring, a screw, and a nut. Due to the long-term effect of the alternating load, the valve with periodic reciprocating motion is more prone to a failure. This paper mainly investigates three kinds of reciprocating compressor valve failures: spring failure, valve plate fracture, and valve plate gap. When the reciprocating compressor valve is abnormal, the performance of the vibration signal in the valve cover side direction will undergo a substantial change. Therefore, this paper extracted valve cover vibration signals as the analysis data.

A vibration-based measurement and analysis technique is an effective measure to monitor and diagnose fault states of machinery due to the abundant operational state information embodied in vibration signals. An experiment on valve failures states for the secondary valve was conducted in real working conditions. Three valve failures states (spring failure, valve plate fracture, and valve plate gap) corresponding to one less spring, the middle valve piece breaks, and a gap on the second and third rings of the outer ring number states simulated with the secondary valve cover side, were tested respectively using a Hubei UT3416 data acquisition instrument and integrated circuit piezoelectric (ICP) acceleration sensors placed on the top of valve surface to collect vibration signals. The position of sensor was marked with a red square in Fig. 7a, and the sample frequency is $5 \mathrm{kHz}$. The vibration signal collected by MATLAB software written programs was analysed. The vibration signals of four primary valve states are shown in Fig. 8 for two periods.

In this paper, the VMD method was employed to decompose the vibration signal of the reciprocating compressor valve faults; the parameters of the VMD method need to be determined, so the default mode number $K=2$ and the bandwidth parameter $\alpha=2000$. In theory, the centre frequency of each 
BLIMF component after the VMD decomposition is distributed from low frequency to high frequency; when the $\mathrm{K}$ value is optimized, the difference between the centre frequency of the $K^{\text {th }}$ BLIMF component and the centre frequency of the $(K-1)^{\text {th }}$ BLIMF component is the biggest. Therefore, the best mode number $K$ value is confirmed using the method of the centre frequency difference maximum. The vibration signals of reciprocating compressor valves are analysed to determine the modal number $K$ value. The vibration signal of valve fracture failure as an example specifically, when $K$ takes different values, the centre frequency of several BLIMF components obtained by the VMD decomposition is shown in Table 1.

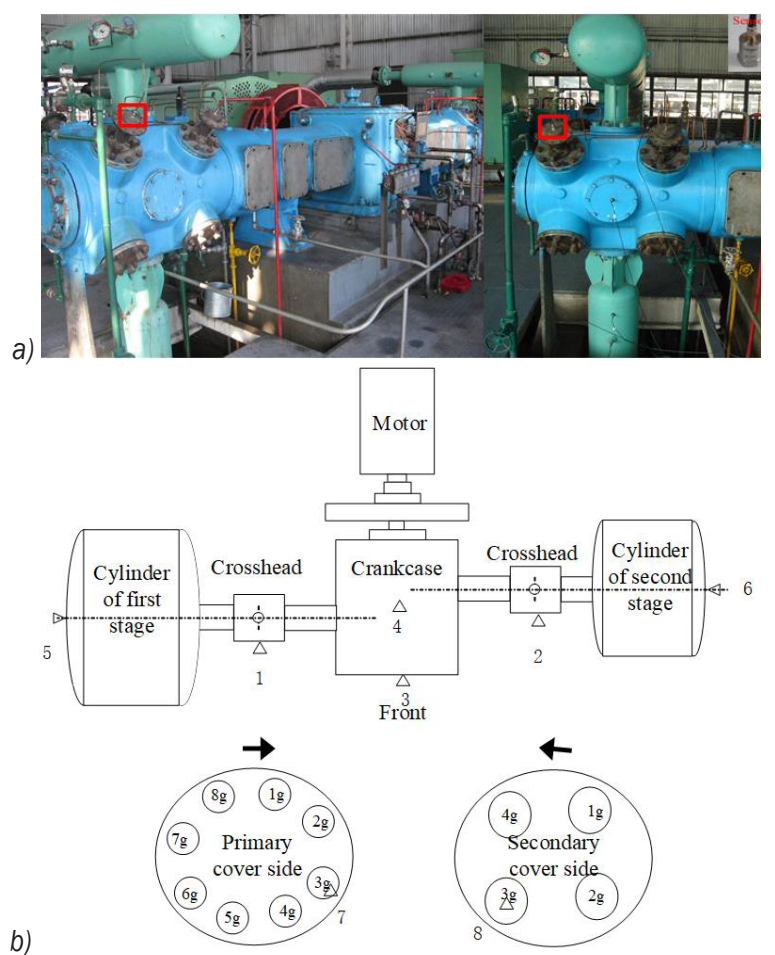

Fig. 7. Two-stage double-acting reciprocating compressor of 2D12 type; a) test bench of reciprocating compressor, b) structural drawing of reciprocating compressor

As can be seen from Table 1, when $K=4$, the difference between the centre frequency of the fourth BLIMF component and the centre frequency of the third BLIMF component is $15953.9 \mathrm{~Hz}$; when $K \geq 5$, the difference between the centre frequency of the $K^{\text {th }}$ BLIMF component and the centre frequency of the $(K-1)^{\text {th }}$ BLIMF component is less than $15953.9 \mathrm{~Hz}$. Therefore, it is considered that when $K=4$, the best value can be obtained. This method is applied to the vibration signals of the four valve fault states, and the
VMD parameters of the vibration signals of the four valve fault states can be obtained, as shown in Table 2 .
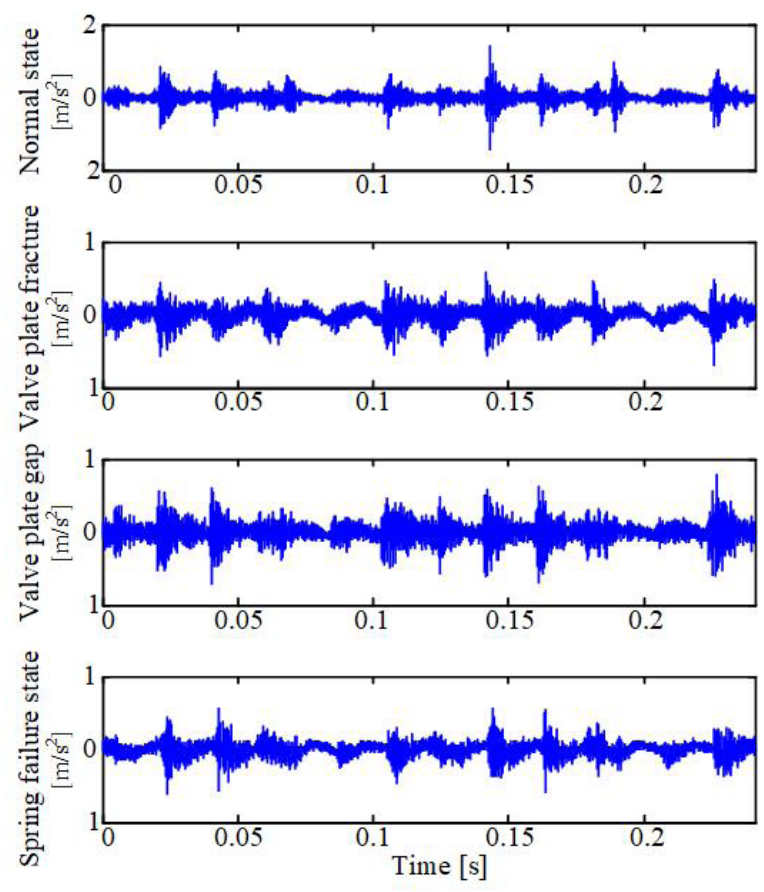

Fig. 8. Vibration acceleration in four reciprocating compressor valve states

Using the VMD parameters in Table 2, the vibration signals of four valve fault states were decomposed by the VMD, then cross correlation coefficient of each BLIMF component in different valve states is respectively calculated in Table 3 . A reasonable threshold is given and the BLIMF component with the main state information is determined to reconstruct signal of each state. The cross-correlation coefficient of the first three BLIMF components of the normal state, valve plate fracture, and valve plate gap is greater than 0.3, and the cross correlation coefficient of the first four BLIMF components of spring failure is greater than 0.4. Therefore, in this paper, the first three BLIMF components of normal state, valve plate fracture, and valve plate gap, and the first four BLIMF components of spring failure were selected for signal reconstruction.

The MMSE values of the reconstruction vibration signals in different valve states were calculated, for which the mode dimension $m=2$, the similar tolerance $r=0.25 \mathrm{SD}$, the maximum scale factor $\tau_{\max }=20$. As shown in Figs. 9 and 10, the MMSE values graphs are the same length of time within the scope of each fault vibration signal. In Figs. 9 and 10, the MMSE values 
Table 1. Center frequency of each BLIMF component of the vibration signal

\begin{tabular}{|c|c|c|c|c|c|c|c|}
\hline & BLIMF1 & BLIMF2 & BLIMF3 & BLIMF4 & BLIMF5 & BLIMF6 & BLIMF7 \\
\hline$K=2$ & 6133.8 & 11668.3 & & & & & \\
\hline$K=3$ & 453.9 & 5865.5 & 8561.9 & & & & \\
\hline$K=4$ & 388.5 & 5510.8 & 7830.2 & 23784.1 & & & \\
\hline$K=5$ & 307.8 & 5080.2 & 6628.6 & 8766.1 & 19442.7 & & \\
\hline$K=6$ & 163.6 & 2979.6 & 5328.9 & 6658.4 & 8787.5 & 23046.0 & \\
\hline$K=7$ & 163.1 & 2977.5 & 5327.6 & 6654.3 & 8762.9 & 20278.8 & 23073.1 \\
\hline
\end{tabular}

of each fault vibration signal decrease progressively with the increasing of scale factor from $\tau=3$, which indicates that the complexity of valve fault vibration signal is decreasing. The MMSE values of the normal state, valve plates fracture, valve plate gap and valve spring failure are represented by MMSE1, MMSE2, MMSE3 and MMSE4, respectively; in general, their size order is MMSE2 > MMSE4 > MMSE3 > MMSE1. However, the MMSE values of the latter part of Fig. 9 and the front part of Fig. 10 appear as overlapping phenomenon. If the calculated MMSE values are directly input into the BTSVM, the accuracy of the fault classification is relatively low. Therefore, the MMSE values need to be further optimized before being input into the BTSVM. The GDE method proposed in this article was used to perform the feature optimization, the assessment curve as shown in Fig. 11.

The sensitivity on the different scales are different, and the larger the scale factor, the lower sensitivity in Fig. 11, so set the threshold condition of $t h>1.00$. According to the definition of conditions, MMSE values of scale factor $\tau$ between 3 and 10 should be selected as a sensitive feature vector subset.

Table 2. VMD parameters of the vibration signals of the four valve fault states

\begin{tabular}{lcc}
\hline Valve states & Modal number $K$ & Bandwidth parameter $\alpha$ \\
\hline Normal state & 4 & 2000 \\
\hline Valve plate fracture & 4 & 2000 \\
\hline Valve plate gap & 4 & 2000 \\
\hline Spring failure & 5 & 2000 \\
\hline
\end{tabular}

Further evaluation is needed to assess the effectiveness of the proposed method based on the MMSE and GDE method, and this paper introduces the binary tree support vector machine (BTSVM) method [26] to [28] to evaluate four valve fault eigenvectors. To test this, 150 eigenvector samples were selected from each valve fault, and 100 were taken as training samples, other 50 as test samples.
For the BTSVM, the radial basis kernel function was employed, and the kernel parameter $\gamma=3.52$ and error penalty parameter $C=1.79$ were optimized with the genetic algorithm; the results are shown in Table 4.

Table 3. Cross correlation coefficient of each BLIMF component in different valve states

\begin{tabular}{lccccc}
\hline \multirow{2}{*}{$\begin{array}{l}\text { Valve } \\
\text { states }\end{array}$} & \multicolumn{5}{c}{ Cross correlation coefficient of each BLIMF component } \\
\cline { 2 - 6 } & BLIMF1 & BLIMF2 & BLIMF3 & BLIMF4 & BLIMF5 \\
\hline $\begin{array}{l}\text { Normal } \\
\text { state }\end{array}$ & 0.3205 & 0.7559 & 0.6192 & 0.2048 & \\
\hline $\begin{array}{l}\text { Valve plate } \\
\text { fracture }\end{array}$ & 0.5808 & 0.7328 & 0.5645 & 0.0790 & \\
\hline $\begin{array}{l}\text { Valve plate } \\
\text { gap }\end{array}$ & 0.4484 & 0.8423 & 0.5185 & 0.1496 & \\
\hline $\begin{array}{l}\text { Spring } \\
\text { failure }\end{array}$ & 0.4928 & 0.4939 & 0.7030 & 0.4935 & 0.0854 \\
\hline
\end{tabular}

To compare the superiority of this fault recognition method, the same number of training and test samples was extracted by other three methods, including the VMD-MMSE and principal component analysis (PCA), the MMSE and GDE, and the VMDMMSE, and then recognized using the BTSVM. The recognition results are also listed in Table 4.

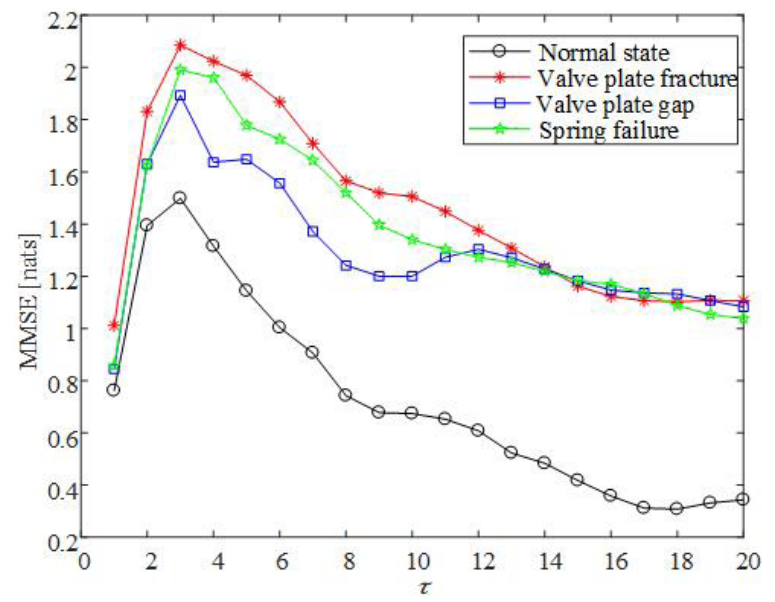

Fig. 9. First MMSE values graphs of the reconstruction vibration signals in different valve states 


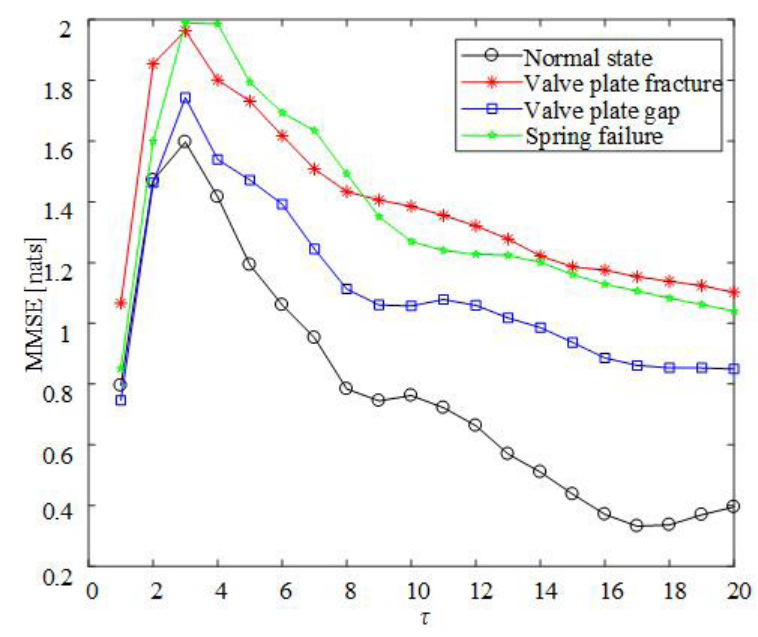

Fig. 10. Second MMSE values graphs of the reconstruction vibration signals in different valve states

Table 4. Recognition accuracy of comparison between different methods

\begin{tabular}{lccccc}
\hline $\begin{array}{l}\text { Feature } \\
\begin{array}{l}\text { extraction } \\
\text { method }\end{array}\end{array}$ & $\begin{array}{c}\text { Normal } \\
\text { state }\end{array}$ & $\begin{array}{c}\text { Spring } \\
\text { failure }\end{array}$ & $\begin{array}{c}\text { Valve } \\
\text { plate } \\
\text { fracture }\end{array}$ & $\begin{array}{c}\text { Valve } \\
\text { plate } \\
\text { gap }\end{array}$ & $\begin{array}{c}\text { Total } \\
\text { accuracy } \\
{[\%]}\end{array}$ \\
\hline $\begin{array}{l}\text { VMD-MMSE } \\
\text { and GDE } \\
\text { method }\end{array}$ & 100 & 98 & 100 & 100 & 99.5 \\
\hline $\begin{array}{l}\text { VMD-MMSE } \\
\text { and PCA }\end{array}$ & 98 & 98 & 98 & 98 & 98 \\
\hline $\begin{array}{l}\text { MMSE and } \\
\text { GDE method }\end{array}$ & 92 & 90 & 92 & 92 & 91.5 \\
\hline VMD-MMSE & 90 & 86 & 86 & 88 & 87.5 \\
\hline
\end{tabular}

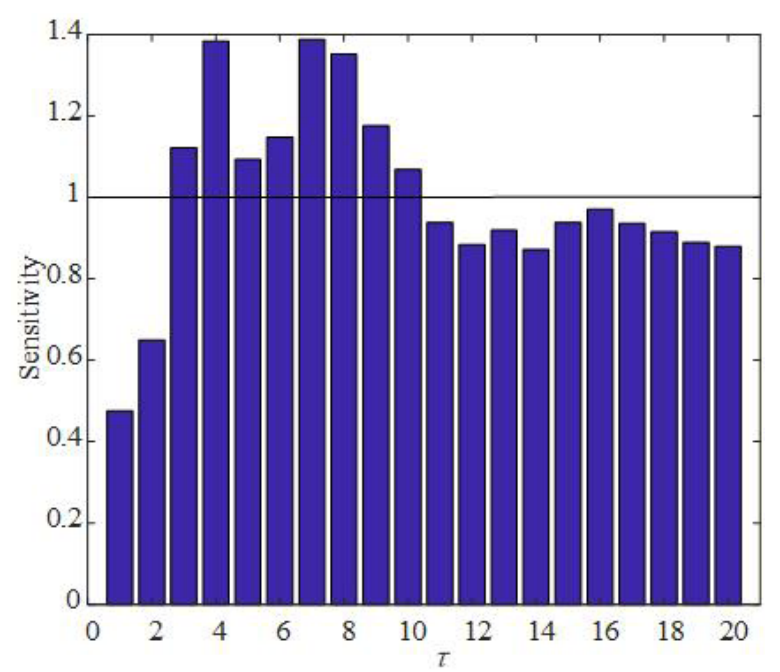

Fig. 11. Assessment curve of reciprocating compressor valve fault

The results show that the accuracy in each state and the total accuracy of the proposed method are better than those of three other feature extraction methods under the condition of the same finite number of samples. From Table 4, we can clearly see that, by comparing the identification results of three methods, the feature vectors extracted by the VMDMMSE and GDE and the VMD-MMSE and PCA are better than those achieved by the MMSE and GDE from the result of recognition rate, so it is necessary to combine the self-decomposition method with the MMSE for resisting noise interference and highlighted information extraction. Furthermore, we also see that the fault recognition rates of the MMSE without the GDE are lower than those of the proposed method, so it validates the necessity of using the GDE in this method. Through the comparative research above, the proposed method is the superior fault recognition method to diagnosis faults of reciprocating compressor valve effectively and accurately.

\section{CONCLUSION}

To satisfy the needs of reciprocating compressor valve fault recognition, from the perspective of fault feature extraction and optimal selection, this paper presents a novel fault diagnosis method based on the modified multiscale entropy and global distance evaluation, and it is applied for fault diagnosis of reciprocating compressor valve faults.

(1) In the proposed method, the VMD method was employed to eliminate the noise interference, which outperforms the traditional time frequency analysis method such as the EMD and Wavelet packet for the reciprocating compressor vibration signals.

(2) After the vibration signal is reconstructed by the VMD method, a novel MMSE method provided with moving-average procedure by replacing mean-average coarse-grained procedure was developed for the vibration signals, which overcomes the obstacle of the conventional MSE yielding undefined entropy or an imprecise estimation of entropy by the large scale factor.

(3) For the appearance of the MMSE eigenvalues overlapping phenomenon, the GDE method was employed to refine the final eigenvector, and it indeed has a higher recognition efficiency and accuracy.

(4) This method was applied for the fault diagnosis of a reciprocating compressor at different valve states, and it demonstrates superior recognition results through the comparison with other feature extraction methods. 
In this paper, the VMD method was used to denoise the vibration signal, the research on the preset decomposition scale has made some progress, and the modal-aliasing phenomenon is suppressed to some extent, but the method of selecting parameters is not adaptive. To understand how to suppress noise more effectively and adaptively suppress modal aliasing, further research is needed.

\section{ACKNOWLEDGEMENTS}

This work was partly supported by the General Financial Grant from the China Postdoctoral Science Foundation (2015M581423), Natural Science Foundation of Heilongjiang Province in China (E2015037, E2016009), and Supported by Northeast Petroleum University Innovation Foundation For Postgraduate (YJSCX2017-020NEPU).

\section{NOMENCLATURES}

\begin{tabular}{|c|c|}
\hline$K$ & initialization modal number, [-] \\
\hline$\sigma$ & penalty factor, $[-]$ \\
\hline$\alpha$ & bandwidth value, $[\mathrm{Hz}]$ \\
\hline$\left\{q_{j}^{(\tau)}\right\}$ & the coarse-grained time series, $[-]$ \\
\hline & length of time series, [-] \\
\hline$\tau$ & time scale factor, $[-]$ \\
\hline$m$ & mode dimension, [-] \\
\hline$r$ & similar tolerance, $[-]$ \\
\hline $\mathrm{Cm}^{m}$ & template matching number, [-] \\
\hline SampEn & sample entropy, [nats] \\
\hline$P_{j}^{(\tau)}$ & $\begin{array}{l}\text { the moving-averaged time series at } \\
\text { a scale factor } \tau,[-]\end{array}$ \\
\hline$T$ & original MMSE feature set, [-] \\
\hline $\operatorname{MMSE}(l, m, n)$ & $\begin{array}{l}\text { sample entropy feature of } l^{\text {th }} \text { scale of } \\
m \text {-category under } n^{\text {th }} \text { sample, [nats] }\end{array}$ \\
\hline $\operatorname{MMSE}(l, m, z)$ & $\begin{array}{l}\text { sample entropy feature of } l \text { th scale of } \\
m \text {-category under } z^{\text {th }} \text { sample, [nats] }\end{array}$ \\
\hline$L$ & number of scale, $[-]$ \\
\hline$M$ & number of state class, [-] \\
\hline$N$ & number of sample, $[-]$ \\
\hline$d_{l, m}$ & $\begin{array}{l}\text { average distance within the class of } \\
\text { similar state samples, [-] }\end{array}$ \\
\hline$D_{l}$ & $\begin{array}{l}\text { average distance class under the } \\
\text { same scale } I \text { of } m \text { classes, [-] }\end{array}$ \\
\hline$D_{l}^{\prime}$ & $\begin{array}{l}\text { average distance between different } \\
\text { class states, }[-]\end{array}$ \\
\hline$M M S E^{\prime}(l, u)$ & $\begin{array}{l}\text { average value of } m \text { samples entropy } \\
\text { under } l^{\text {th }} \text { scale of } u^{\text {th }} \text { class, [nats] }\end{array}$ \\
\hline$M M S E^{\prime}(l, v)$ & $\begin{array}{l}\text { average value of } \mathrm{m} \text { samples entropy } \\
\text { under } l^{\text {th }} \text { scale of vth class, [nats] }\end{array}$ \\
\hline$\varphi_{l}$ & $\begin{array}{l}\text { the } l^{\text {th }} \text { scale feature of sensitivity of } \\
\text { classifying } M \text { class, [-] }\end{array}$ \\
\hline
\end{tabular}

\author{
$\varphi_{\text {mean }} \quad$ average sensitivity, [-] \\ th the threshold, [-] \\ $\tau_{\max } \quad$ the maximum scale factor, [-] \\ $\gamma \quad$ kernel parameter, [-] \\ $C \quad$ error penalty parameter, [-]
}

\section{REFERENCES}

[1] Jin, G., Yang, S. (1991). Compressor Reliability. Mechanical Industry Press, Beijing. (in Chinese)

[2] Pincus, S.M. (1991) Approximate entropy as a measure of system complexity. Proceedings of the National Academy of Sciences, vol. 88, no. 6, p. 2297-2301, Dol:10.1073/ pnas.88.6.2297.

[3] Richman, J.S., Moorman, J.R. (2000). Physiological timeseries analysis using approximate entropy and sample entropy. American Journal of Physiology Heart Circulatory Physiology, vol. 278, no. 6, p. 2039-2049, Dol:10.1152/ ajpheart.2000.278.6.H2039.

[4] Costa, M., Goldberger, A.L., Peng, C.-K. (2005). Multiscale entropy analysis of biological signals. Physical Review E, vol. 71, no. 2, p. 1-18, Dol:10.1103/PhysRevE.71.021906.

[5] Costa, M., Goldberger, A.L., Peng, C.-K. (2002) Multiscale entropy analysis of complex physiologic time series. Physical Review Letters, vol. 89, no. 6, p. 1-18, D0l:10.1103/ PhysRevLett.89.068102.

[6] Qi, Z., Xu, Z., Lu, Z. (2008). Application of approximate entropy in engine fault diagnosis. Journal of Ordnance Engineering College, vol. 20, no. 2, p. 39-42. (in Chinese)

[7] Zhao, H., Wang, J., Xing, J., Gao, Y. (2015). A feature extraction method based on LMD and MSE and its application for fault diagnosis of reciprocating compressor. Journal of Vibroengineering, vol. 17, no. 7, p. 3515-3526.

[8] Wang, Z., Yao, D., Duan, L. (2007). Study on the method of oil field reciprocating compressor valve fault diagnosis based on fuzzy clustering. Mechanical Strength, vol. 29, no. 3, p. 521524. (in Chinese)

[9] Figlus, T., Gnap, J. Skrúcany,T. Šarkan, B., Stoklosa, J. (2016). The use of denoising and analysis of the acoustic signal entropy in diagnosing engine valve clearance. Entropy, vol. 18, no. 7, p. 1-11, D0I:10.3390/e18070253.

[10] Geng, Z., Chen, J. (2005). Investigation into piston-slapinduced vibration for engine condition simulation and monitoring. Journal of Sound and Vibration, vol. 282, no. 3-5, p. 735-775, D0l:10.1016/j.jsv.2004.03.057.

[11] Jedlinski, L., Caban, J., Krzywonos, L., Wierzbicki, S., Brumercik, F. (2015). Application of vibration signal in the diagnosis of IC engine valve clearance. Journal of Vibroengineering, vol. 17, no. 1, p. 175-187.

[12] Zhao, H., Wang, J., Han, H., Gao, Y. (2016). A feature extraction method based on HLMD and MFE for bearing clearance fault of reciprocating compressor. Measurement, vol. 89, p. 34-43, D0l:10.1016/j.measurement.2016.03.076.

[13] Zhao, H., Wang, J., Jay, L., Li, Y. (2018). A compound interpolation envelope local mean decomposition and its application for fault diagnosis of reciprocating compressors. Mechanical Systems and Signal Processing, vol. 110, p. 273295, DOI:10.1016/j.ymssp.2018.03.035. 
[14] Li, Y., Li, G., Yang, Y., Liang, X., Xu, M. (2018). A fault diagnosis scheme for planetary gearboxes using adaptive multi-scale morphology filter and modified hierarchical permutation entropy. Mechanical Systems and Signal Processing, vol. 105, p. 319-337, DOl:10.1016/J.ymssp.2017.12.008.

[15] Li, Z., Jiang, Y., Hu, C., Peng, Z. (2016). Recent progress on decoupling diagnosis of hybrid failures in gear transmission systems using vibration sensor signal: A review. Measurement, vol. 90, p. 4-19, D0I:10.1016/j.measurement.2016.04.036.

[16] Zhang, C., Peng, Z., Chen, S., Li, Z., Wang, J. (2018). A gearbox fault diagnosis method based on frequency-modulated empirical mode decomposition and support vector machine. Proceedings of the Institution of Mechanical Engineers, Part C: Journal of Mechanical Engineering Science, vol. 232, no. 2, p. 369-380, DOI:10.1177/0954406216677102.

[17] Bi, F., Li, L., Zhang, J., Ma, T. (2015). Source identification of gasoline engine noise based on continuous wavelet transform and EEMD-RobustICA. Applied Acoustic, vol. 100, p. 34-42, DOI:10.1016/J.apacoust.2015.07.007.

[18] Li, N., Yang, J., Zhou, R., Liang, C. (2016). Determination of knock characteristics in spark ignition engines: an approach based on ensemble empirical mode decomposition. Measurement Science and Technology, vol. 27, no. 4, p. 045109, D0I:10.1088/0957-0233/27/4/045109.

[19] Dragomiretskiy, K., Zosso, D. (2014). Variational mode decomposition. IEEE Transaction on Signal Process, vol. 62, no. 3, p. 531-544, DOl:10.1109/TSP.2013.2288675.

[20] Wang, Y., Markert. R, Xiang, J., Zheng, W. (2015). Research on variational mode decomposition and its application in detecting rub-impact fault of rotor system. Mechanical Systems and Signal Processing, vol. 60-61, p. 243-251, D0l:10.1016/j.ymssp.2015.02.020.

[21] Liu, Y., Wang, J., Li, Y., Zhao, H., Chen, S. (2017). Feature extraction method based on VMD and MFDFA for fault diagnosis of reciprocating compressor Valve. Journal of Vibroengineering, vol. 19, no. 8, p. 6007-6020, Dol:10.21595/ jve.2017.18726.

[22] An, X., Yang, J. (2016). Denoising of hydropower unit vibration signal based on variational mode decomposition and approximate entropy. Transaction of the Institute of Measurement abd Control, vol. 38, no. 3, p. 282-292, DOI:10.1177/0142331215592064.

[23] Li, Y., Li, G., Wei, Y., Liu, B., Liang, X. (2018). Health condition identification of planetary gearboxes based on variational mode decomposition and generalized composite muliscale symbolic dynamic entropy. ISA Transactions, vol. 81, p. 329341, D0I:10.1016/j.isatra.2018.06.001.

[24] Upadhyay, A., Pachori, R.B. (2015). Instantaneous voiced/nonvoiced detection in speech signals based on variational mode decomposition. Journal of the Franklin Institute, vol. 352, no. 7, p. 2679-2707, D0l:10.1016/j.jfranklin.2015.04.001.

[25] Lahmiri, S. (2015). Long memory in international financial markets trends and short movements during 2008 financial crisis based on variational mode decomposition and detrended fluctuation analysis. Physica A: Statistical Mechanics and it Applications, vol. 437, p. 130-138, DOl:10.1016/j.physa.2015.05.067.

[26] Wilk-Kolodziejczyk, D., Regulski, K., Gumienny, G. (2016). Comparative analysis of the properties of the nodular cast iron with carbides and the austempered ductile iron with use of the machine learning and the support vector machine. International Journal of Advanced Manufacturing Technology, vol. 87, no. 1-4, p. 1077-1093, DOl:10.1007/s00170-0168510-y.

[27] Wilk-Kolodziejczyk, D., Regulski, K., Gumienny, G., Kacprzyk, B., Kluska-Nawarecka, S., Jaskowiec, K. (2018). Data mining tools in identifying the components of the microstructure of compacted graphite iron based on the content of alloying elements. International Journal of Advanced Manufacturing Technology, vol. 95, no. 9-12, p. 3127-3139, D0l:10.1007/ s00170-017-1430-7.

[28] Li, Y., Yang, Y., Wang, X., Liu, B., Liang, X. (2018). Early fault diagnosis of rolling bearings based on hierarchical symbol dynamic entropy and binary tree support vector machine. Journal of Sound and Vibration, vol. 428, p. 72-86, DOI:10.1016/j.jsv.2018.04.036. 